\title{
Single Oral Dose Toxicity Study of Pinelliae Rhizoma Aqueous Extract in ICR Mice
}

\author{
Young-Kwon Lim ${ }^{1}$, Ji-Ha Park', Bu-ll Seo ${ }^{1}$, Seong-Soo Roh ${ }^{1}$ and Sae-Kwang Ku ${ }^{2,3}$ \\ ${ }^{1}$ Department of Herbalogy, College of Oriental Medicine, \\ ${ }^{2}$ Department of Anatomy and Histology, College of Oriental Medicine, \\ ${ }^{3}$ Development Team for The New Drug of Oriental Medicine (BK21 program), \\ Daegu Haany University, Gyeongsan 712-715, Korea
}

(Received May 13, 2009; Accepted August 1, 2009)

\begin{abstract}
This study was conducted to obtain acute information of the oral dose toxicity of lyophilized water extract of Pinelliae Rhizoma, a dried tuber of Pinellia ternata (PR) in male and female mice. In order to calculated $50 \%$ lethal dose $\left(L_{50}\right)$ and approximate lethal dose (ALD), test material was once orally administered to male and female ICR mice at dose levels of 2000, 1000, 500, 250, 125 and 0 (vehicle control) $\mathrm{ml} / \mathrm{kg}$ (body weight). The mortality and changes in body weight, clinical signs, gross observation, organ weight and histopathology of principle organs were monitored 14 days after treatment with PR extract. We could not find any mortalities, clinical signs, changes in the body and organ weights, gross and histopathological findings except for dose-dependent increases in the hepatic fatty change frequencies detected in PR extract 2000 and $1000 \mathrm{mg} / \mathrm{kg}$ treated in both male and female mice. The results obtained in this study suggest that $L D_{50}$ and approximate $L D$ in mice after single oral dose of PR extracts were considered over $2000 \mathrm{mg} / \mathrm{kg}$ in both and female male mice, but more than $1000 \mathrm{mg} / \mathrm{kg}$ of PR extracts treatment could induce slight hepatotoxicity the fatty changes in mice.
\end{abstract}

Key words: Pinelliae Rhizoma, Pinellia ternata, Single oral dose toxicity, Mice, Histopathology

\section{INTRODUCTION}

A traditional Korean herbal medicine, Pinelliae Rhizoma (PR) is a dried tuber of Pinellia ternata (Thunberg) Breitenbach (Family: Araceae), and has been used as sedative and antiemesis agents for pregnant (Ho and Liu, 1975; Maki et al., 1987; Lee et al., 1993; Kurata et al., 1998). Until now, various pharmacological effects of PR have been revealed; anti-cancer effect (Li et al., 2007), anti-obesity effect (Kim et al., 2006), emetic effect (Zhao et al., 2005), anti-emetic effect (Kurata et al., 1998) and neuroprotective effect (Shi et al., 1994). In addition, the therapeutic effects on hepatitis B infections (Chen et al., 2005), neuroprotective effect (Koo et al., 2005; Chung et al., 2006), anti-stress effect (Katagiri et al., 2004) and gastric profusion effect (Chen et al., 2002) of mixed herbal formula containing

Correspondence to: Sae-Kwang Ku, Department of Anatomy and Histology, College of Oriental Medicine, Daegu Haany University, 290, Yugok-dong, Gyeongsan-si, Gyeongsangbukdo 712-715, Korea

E-mail: gucci200@hanmail.net
PR extracts also have been researched.

As increase of the concern in the functional food and well being in life, the demands and consumption of functional food originated from natural sources are increased (Lee et al., 2003). However, the toxicological aspects about these natural origin-functional foods has been neglected because of the reasons that they has been used as various purpose for long times. Therefore, it is considered that more detail and systemic toxicological studies should be tested for control the abuse and potential toxicities even if they have been used as traditional folk medicine. Although it has been regarded that $\mathrm{PR}$ is a representative toxic irritable herbs in Korean medicine (Park and Seo, 2000), the scientific toxicological studies about PR extracts have not conducted except for 4 weeks repeated oral dose toxicity test in rats (Lee et al., 2003), cytotoxicity (Lee et al., 1993) and some poster presentational gentoxicity (Yang et al., 1990). There are no repots dealing the toxicological aspects of PR water extracts in mouse, even if the basic single dose toxicities.

The objective of the present study, therefore, was to 
obtain the primary safety information about PR extracts, lyophilized water extract of Pinellia ternata and further clarifies their safety for clinical use. In order to observe the $50 \%$ lethal dose $\left(\mathrm{LD}_{50}\right)$, approximate lethal dosage (ALD), test articles were once orally administered to female and male ICR mice at dose levels of 2000, 1000, 500 and 0 (control) $\mathrm{mg} / \mathrm{kg}$ (body weight) according to the recommendation of KFDA Guidelines (2005-60, 2005). The mortality and changes on body weight, clinical signs and gross observation were monitored during 14 days after oral administration of PR extracts according to Korea Food and Drug Administration (KFDA) Guidelines (2005-60, 2005) with organ weights and histopathology of 12 types of principle organs.

\section{MATERIALS AND METHODS}

Experimental animals. Each of twenty female and male ICR mice (6-wk old upon receipt, SLC, Japan) was used after acclimatization for 7 days. Five animals were allocated per a polycarbonate cage in a temperature $\left(20 \sim 25^{\circ} \mathrm{C}\right)$ and humidity (40 45\%) controlled room. Light : dark cycle was $12 \mathrm{~h}: 12 \mathrm{~h}$ and feed (Samyang, Korea) and water were supplied free to access. All animals were overnight fasted (about $18 \mathrm{~h}$ ) before dosing and terminal necropsy. Animals were marked by picric acid.

Preparation of PR extracts. Aqueous PR extracts (absorption rate $29.36 \%$ ) were prepared by routine methods using rotary vacuum evaporator $(\mathrm{BUCHI}$ Rotavapor R-144, Switzerland) and programmable freeze dryer (IIShin Lab., Korea) from PR, which were purchased from local voucher (Cho-Heung Pharmaceutical Ind. Co. (Daegu, Korea) after confirm the morphology under microscopy. Powders of PR extracts were light brown powder. PR extracts were stored in a refrigerator at $-20^{\circ} \mathrm{C}$ to protect from light and degeneration. The appearance of PR extracts in vehicle was clear light brown solution in distilled water and it is well soluble upto $200 \mathrm{mg} / \mathrm{ml}$ concentration levels. The test article was single orally administered at a dosage volume of $20 \mathrm{ml} / \mathrm{kg}$ using distilled water as vehicle.

Grouping and dosing. The animals were distributed into 8 groups 5 mice per group upon receipt. PR extracts have been used as folk medicine and ingredients of medicinal food for long times and no revealed toxicological data was available, the highest dosage level was selected as $2000 \mathrm{mg} / \mathrm{kg}$ according to the recommended by KFDA $(2005-60,2005)$ and Organization for Economic Co-Operation and Development (OECD) (2001) guidelines, the limited dosages, and 1000 and $500 \mathrm{mg} / \mathrm{kg}$ was selected using common ratio 2 . In addition, a vehicle control group was added. Animal was once orally dosed using a sonde attached to a syringe of $1 \mathrm{ml}$ after overnight fasting (about 18hr, water was not restricted). Feed and water were restricted further for about $3 \mathrm{~h}$.

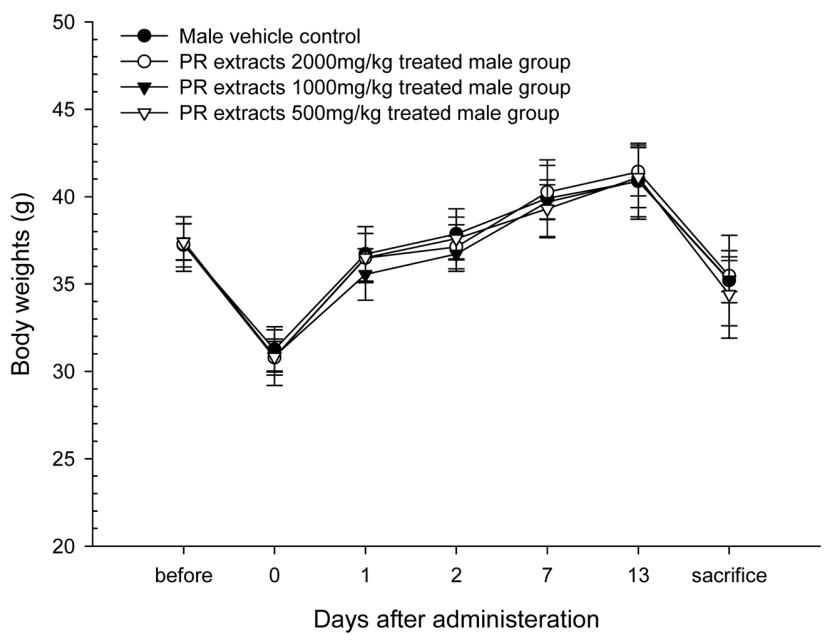

Fig. 1. Body weight changes in male mice after once oral dose of PR extract. No meaningful changes were detected in all PR extract treated groups as compared with vehicle control. Before means 1 day before administration; Day 0 means at administration; All animals at sacrifice and Day 0 overnight fasted.

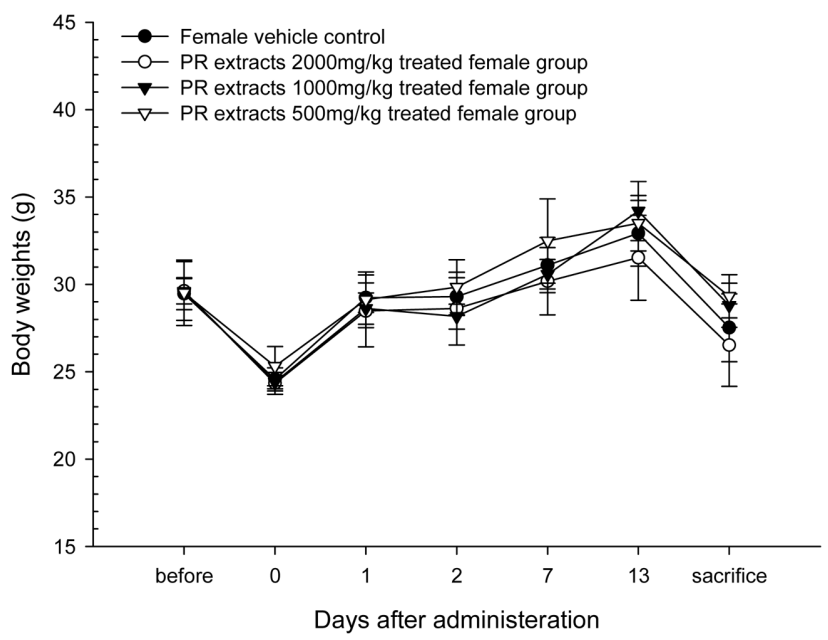

Fig. 2. Body weight changes in female mice after single oral dose of PR extract. No meaningful changes were detected in all PR extract treated groups as compared with vehicle control. Before means 1 day before administration; Day 0 means at administration; All animals at sacrifice and Day 0 overnight fasted. 
Observation of clinical signs. All abnormal clinical signs were recorded before and after dosing at least twice a day based on the functional observational battery test (Irwin, 1968; Dourish, 1987).

Body weight changes. Body weights were measured at the day of dosing (Day 0 ) immediately before treatment, 1, 2, 7, 13 and 14 days after dosing. In addition, to reduce the individual body weight differences of animals at initial dosing, body weigh gains during Day 0 Day 7, Day 7 Day 13 and Day 0 Day 14 were also calculated based on measured body weight at each day.

Necropsy. All unscheduled died animals were grossly observed immediately after finding them and all survived animals were subjected to terminal necropsy. Animals were asphyxiated by carbon dioxide and gross necropsy was performed in all animals at Day 14 after overnight fasting (about $18 \mathrm{~h}$, water was not restricted).

Specific organs grossly observed: lung, heart, kidney, spleen, testis, liver, pancreas, epididymis, popliteal lymph node, ovary, brain, and uterus.

Organ weight measurement. The absolute organ weight was measured and then relative organ weight (\% of body weight) was calculated for the following organs of all experimental animals when they were sacrificed.

Measured organs: lung, heart, kidney (left), spleen, testis (left), liver, pancreas (splenic lobes), epididymis (left), popliteal lymph node (left), ovary (left), brain, and uterus.

Histopathology. Principle organs listed below were sampled at terminal necropsy, and fixed in $10 \% \mathrm{NBF}$ (neutral buffered formalin). After $18 \mathrm{~h}$ of fixation, paraffin embedding was conducted and $3 \sim 4 \mu \mathrm{m}$ sections were

Table 1. Body weight $(\mathrm{g})$ gains after oral treatment of $P R$ extracts

\begin{tabular}{llll}
\hline \hline \multirow{2}{*}{ Group } & \multicolumn{3}{c}{ Intervals } \\
\cline { 2 - 4 } & Day 0 Day 7 Day 7 Day 13 Day 0 Day 14 \\
\hline Male & & & \\
Vehicle control & $8.64 \pm 1.25$ & $0.98 \pm 0.84$ & $3.94 \pm 1.83$ \\
$2000 \mathrm{mg} / \mathrm{kg}$ & $9.48 \pm 1.75$ & $1.16 \pm 0.85$ & $4.68 \pm 1.07$ \\
$1000 \mathrm{mg} / \mathrm{kg}$ & $8.82 \pm 0.78$ & $1.22 \pm 1.32$ & $4.38 \pm 1.08$ \\
$500 \mathrm{mg} / \mathrm{kg}$ & $8.48 \pm 1.26$ & $1.80 \pm 0.51$ & $3.58 \pm 2.24$ \\
\hline Female & & & \\
Vehicle control & $3.90 \pm 1.83$ & $1.76 \pm 0.85$ & $2.96 \pm 2.07$ \\
$2000 \mathrm{mg} / \mathrm{kg}$ & $3.24 \pm 1.57$ & $2.28 \pm 1.40$ & $3.50 \pm 1.07$ \\
$1000 \mathrm{mg} / \mathrm{kg}$ & $4.32 \pm 0.43$ & $1.24 \pm 0.59$ & $2.14 \pm 1.15$ \\
$500 \mathrm{mg} / \mathrm{kg}$ & $5.26 \pm 1.82$ & $0.96 \pm 1.63$ & $3.72 \pm 1.22$ \\
\hline
\end{tabular}

Values are expressed as mean \pm S.D., $g(n=5)$; ${ }^{a}$ Day of treatment.

Table 2. Changes on the absolute organ weights $(\mathrm{g})$ after oral treatment of PR extracts

\begin{tabular}{|c|c|c|c|c|c|c|c|c|c|c|c|c|}
\hline \multirow[b]{2}{*}{ Group } & \multicolumn{12}{|c|}{ Organs: Male } \\
\hline & Lung & Heart & Thymus & $\begin{array}{c}\text { Kidney } \\
\mathrm{L}\end{array}$ & $\begin{array}{l}\text { Adrenal } \\
\text { gland L }\end{array}$ & Spleen & Testis L & Liver & $\begin{array}{c}\text { Pancreas } \\
S\end{array}$ & Brain & $\begin{array}{c}\text { Epididymis } \\
L\end{array}$ & $\begin{array}{l}\text { Lymph } \\
\text { node } L^{a}\end{array}$ \\
\hline $\begin{array}{l}\text { Vehicle } \\
\text { control }\end{array}$ & $\begin{array}{c}0.215 \pm \\
0.007\end{array}$ & $\begin{array}{c}0.179 \pm \\
0.011\end{array}$ & $\begin{array}{c}0.062 \pm \\
0.013\end{array}$ & $\begin{array}{c}0.307 \pm \\
0.048\end{array}$ & $\begin{array}{c}0.008 \pm \\
0.002\end{array}$ & $\begin{array}{c}0.113 \pm \\
0.027\end{array}$ & 0.013 & $\begin{array}{c}1.777 \pm \\
0.179\end{array}$ & $\begin{array}{c}0.185 \pm \\
0.027\end{array}$ & $\begin{array}{c}0.484 \pm \\
0.017\end{array}$ & $\begin{array}{c}0.060 \pm \\
0.012\end{array}$ & $\begin{array}{c}0.012 \pm \\
0.003\end{array}$ \\
\hline $\begin{array}{l}2000 \\
\mathrm{mg} / \mathrm{kg}\end{array}$ & $\begin{array}{c}0.213 \pm \\
0.022\end{array}$ & $\begin{array}{c}0.178 \pm \\
0.013\end{array}$ & $\begin{array}{c}0.058 \pm \\
0.006\end{array}$ & $\begin{array}{c}0.305 \pm \\
0.031\end{array}$ & $\begin{array}{c}0.006 \pm \\
0.002\end{array}$ & $\begin{array}{c}0.117 \pm \\
0.009\end{array}$ & $\begin{array}{c}0.127 \pm \\
0.017\end{array}$ & $\begin{array}{c}1.839 \pm \\
0.108\end{array}$ & $\begin{array}{c}0.215 \pm \\
0.032\end{array}$ & $\begin{array}{c}0.494 \pm \\
0.017\end{array}$ & & $\begin{array}{c}0.009 \pm \\
0.004\end{array}$ \\
\hline $\begin{array}{l}1000 \\
\mathrm{mg} / \mathrm{kg}\end{array}$ & $\begin{array}{c}0.210 \pm \\
0.015\end{array}$ & $\begin{array}{c}0.187 \pm \\
0.014\end{array}$ & $\begin{array}{c}0.052 \pm \\
0.012\end{array}$ & $\begin{array}{c}0.322 \pm \\
0.022\end{array}$ & $\begin{array}{c}0.008 \pm \\
0.004\end{array}$ & $\begin{array}{c}0.103 \pm \\
0.015\end{array}$ & $\begin{array}{c}0.118 \pm \\
0.012\end{array}$ & $\begin{array}{c}1.833 \pm \\
0.096\end{array}$ & $\begin{array}{c}0.193 \pm \\
0.019\end{array}$ & $\begin{array}{c}0.487 \pm \\
0.013\end{array}$ & $\begin{array}{c}0.047 \pm \\
0.007\end{array}$ & $\begin{array}{c}0.012 \pm \\
0.003\end{array}$ \\
\hline \multirow[t]{3}{*}{$\begin{array}{l}500 \\
\mathrm{mg} / \mathrm{kg}\end{array}$} & $\begin{array}{c}0.208 \pm \\
0.021\end{array}$ & $\begin{array}{c}0.183 \pm \\
0.015\end{array}$ & $\begin{array}{c}0.033 \pm \\
0.016^{*}\end{array}$ & $\begin{array}{c}0.328 \pm \\
0.051\end{array}$ & & $\begin{array}{c}0.088 \pm \\
0.013\end{array}$ & $\begin{array}{c}0.123 \pm \\
0.011\end{array}$ & $\begin{array}{c}1.788 \pm \\
0.346\end{array}$ & & & & \\
\hline & \multicolumn{12}{|c|}{ Organs: Female } \\
\hline & Lung & Heart & Thymus & $\begin{array}{c}\text { Kidney } \\
\mathrm{L}\end{array}$ & $\begin{array}{l}\text { Adrenal } \\
\text { gland L }\end{array}$ & Spleen & $\begin{array}{c}\text { Ovary } \\
L\end{array}$ & Liver & $\begin{array}{c}\text { Pancreas } \\
\text { S }\end{array}$ & Brain & Jterus & \\
\hline $\begin{array}{l}\text { Vehicle } \\
\text { control }\end{array}$ & $\begin{array}{c}0.193 \pm \\
0.009\end{array}$ & $\begin{array}{c}0.149 \pm \\
0.011\end{array}$ & $\begin{array}{c}0.059 \pm \\
0.008\end{array}$ & $\begin{array}{c}0.211 \pm \\
0.020\end{array}$ & $\begin{array}{c}0.007 \pm \\
0.003\end{array}$ & $\begin{array}{c}0.097 \pm \\
0.017\end{array}$ & $\begin{array}{c}0.023 \pm \\
0.006\end{array}$ & $\begin{array}{c}1.312 \pm \\
0.149\end{array}$ & $\begin{array}{c}0.165 \pm \\
0.013\end{array}$ & $\begin{array}{c}0.484 \pm \\
0.021\end{array}$ & $\begin{array}{c}0.205 \pm \\
0.027\end{array}$ & $\begin{array}{c}0.013 \pm \\
0.002\end{array}$ \\
\hline $\begin{array}{l}2000 \\
\mathrm{mg} / \mathrm{kg}\end{array}$ & $\begin{array}{c}0.192 \pm \\
0.017\end{array}$ & $\begin{array}{c}0.144 \pm \\
0.012\end{array}$ & $\begin{array}{c}0.055 \pm \\
0.012\end{array}$ & $\begin{array}{c}0.205 \pm \\
0.014\end{array}$ & $\begin{array}{c}0.006 \pm \\
0.003\end{array}$ & $\begin{array}{c}0.098 \pm \\
0.039\end{array}$ & $\begin{array}{c}0.021 \pm \\
0.004\end{array}$ & $\begin{array}{c}1.259 \pm \\
0.163\end{array}$ & $\begin{array}{c}0.151 \pm \\
0.027\end{array}$ & $\begin{array}{c}0.472 \pm \\
0.012\end{array}$ & $\begin{array}{c}0.161 \pm \\
0.056\end{array}$ & $\begin{array}{c}0.011 \pm \\
0.005\end{array}$ \\
\hline $\begin{array}{l}1000 \\
\mathrm{mg} / \mathrm{kg}\end{array}$ & $\begin{array}{c}0.198 \pm \\
0.026\end{array}$ & $\begin{array}{c}0.154 \pm \\
0.009\end{array}$ & $\begin{array}{c}0.067 \pm \\
0.011\end{array}$ & $\begin{array}{c}0.220 \pm \\
0.014\end{array}$ & $\begin{array}{c}0.008 \pm \\
0.004\end{array}$ & $\begin{array}{c}0.122 \pm \\
0.036\end{array}$ & $\begin{array}{c}0.024 \pm \\
0.009\end{array}$ & $\begin{array}{c}1.517 \pm \\
0.132\end{array}$ & $\begin{array}{c}0.184 \pm \\
0.022\end{array}$ & $\begin{array}{c}0.469 \pm \\
0.016\end{array}$ & $\begin{array}{c}0.204 \pm \\
0.047\end{array}$ & $\begin{array}{c}0.011 \pm \\
0.002\end{array}$ \\
\hline $\begin{array}{l}500 \\
\mathrm{mg} / \mathrm{kg}\end{array}$ & $\begin{array}{c}0.192 \pm \\
0.015\end{array}$ & $\begin{array}{c}0.145 \pm \\
0.016\end{array}$ & $\begin{array}{c}0.061 \pm \\
0.010\end{array}$ & $\begin{array}{c}0.208 \pm \\
0.031\end{array}$ & $\begin{array}{c}0.007 \pm \\
0.004\end{array}$ & $\begin{array}{c}0.114 \pm \\
0.013\end{array}$ & $\begin{array}{c}0.028 \pm \\
0.005\end{array}$ & $\begin{array}{c}1.386 \pm \\
0.163\end{array}$ & $\begin{array}{c}0.175 \pm \\
0.021\end{array}$ & $\begin{array}{c}0.470 \pm \\
0.019\end{array}$ & $\begin{array}{c}0.152 \pm \\
0.050\end{array}$ & $\begin{array}{c}0.011 \pm \\
0.003\end{array}$ \\
\hline
\end{tabular}

Values are expressed as mean \pm S.D., organ weight $(\mathrm{g})(\mathrm{n}=5)$; $\mathrm{L}$, left sides; S, splenic lobes; ${ }^{\mathrm{a}}$ Popliteal lymph node; * $p<0.05$ as compared with vehicle control. 
prepared by routine histological methods. Representative sections of each specified organs were stained with Hematoxylin \& Eosin for light microscopical examination.
Specific organs sampled: lung, heart, kidney (left), spleen, testis (left), liver, pancreas (splenic lobes), epididymis (left), popliteal lymph node (left), ovary (left),

Table 3. Changes on the relative organ weights $(\mathrm{g})$ after oral treatment of PR extracts

\begin{tabular}{|c|c|c|c|c|c|c|c|c|c|c|c|c|}
\hline \multirow[b]{2}{*}{ Group } & \multicolumn{12}{|c|}{ Organs: Male } \\
\hline & Lung & Heart & Thymus & $\begin{array}{c}\text { Kidney } \\
\mathrm{L}\end{array}$ & $\begin{array}{l}\text { Adrenal } \\
\text { gland L }\end{array}$ & Spleen & $\begin{array}{c}\text { Testis } \\
\mathrm{L}\end{array}$ & Liver & $\begin{array}{l}\text { ancreas } \\
S\end{array}$ & Brain & $\begin{array}{l}\text { Epididymis } \\
\mathrm{L}\end{array}$ & \\
\hline $\begin{array}{l}\text { Vehicle } \\
\text { control }\end{array}$ & $\begin{array}{c}0.193 \pm \\
0.009\end{array}$ & $\begin{array}{c}0.149 \pm \\
0.011\end{array}$ & $\begin{array}{c}0.059 \pm \\
0.008\end{array}$ & $\begin{array}{c}0.211 \pm \\
0.020\end{array}$ & $\begin{array}{c}0.007 \pm \\
0.003\end{array}$ & $\begin{array}{c}0.097 \pm \\
0.017\end{array}$ & $\begin{array}{c}0.023 \pm \\
0.006\end{array}$ & $\begin{array}{c}1.312 \pm \\
0.149\end{array}$ & $\begin{array}{c}0.165 \pm \\
0.013\end{array}$ & $\begin{array}{c}0.484 \pm \\
0.021\end{array}$ & $\begin{array}{c}0.205 \pm \\
0.027\end{array}$ & $\begin{array}{c}0.013 \pm \\
0.002\end{array}$ \\
\hline $\begin{array}{l}2000 \\
\mathrm{mg} / \mathrm{kg}\end{array}$ & $\begin{array}{c}0.192 \pm \\
0.017\end{array}$ & $\begin{array}{c}0.144 \pm \\
0.012\end{array}$ & $\begin{array}{c}0.055 \pm \\
0.012\end{array}$ & $\begin{array}{c}0.205 \pm \\
0.014\end{array}$ & $\begin{array}{c}0.006 \pm \\
0.003\end{array}$ & $\begin{array}{c}0.098 \pm \\
0.039\end{array}$ & $\begin{array}{c}0.021 \pm \\
0.004\end{array}$ & $\begin{array}{c}1.259 \pm \\
0.163\end{array}$ & $\begin{array}{c}0.151 \pm \\
0.027\end{array}$ & $\begin{array}{c}0.472 \pm \\
0.012\end{array}$ & $\begin{array}{c}0.161 \pm \\
0.056\end{array}$ & $\begin{array}{c}0.011 \pm \\
0.005\end{array}$ \\
\hline $\begin{array}{l}1000 \\
\mathrm{mg} / \mathrm{kg}\end{array}$ & $\begin{array}{c}0.198 \pm \\
0.026\end{array}$ & $\begin{array}{c}0.154 \pm \\
0.009\end{array}$ & $\begin{array}{c}0.067 \pm \\
0.011\end{array}$ & $\begin{array}{c}0.220 \pm \\
0.014\end{array}$ & $\begin{array}{c}0.008 \pm \\
0.004\end{array}$ & $\begin{array}{c}0.122 \pm \\
0.036\end{array}$ & $\begin{array}{c}0.024 \pm \\
0.009\end{array}$ & $\begin{array}{c}1.517 \pm \\
0.132\end{array}$ & $\begin{array}{c}0.184 \pm \\
0.022\end{array}$ & $\begin{array}{c}0.469 \pm \\
0.016\end{array}$ & $\begin{array}{c}0.204 \pm \\
0.047\end{array}$ & $\begin{array}{c}0.011 \pm \\
0.002\end{array}$ \\
\hline \multirow[t]{3}{*}{$\begin{array}{l}500 \\
\mathrm{mg} / \mathrm{kg}\end{array}$} & $\begin{array}{c}0.192 \pm \\
0.015\end{array}$ & $\begin{array}{c}0.145 \pm \\
0.016\end{array}$ & $\begin{array}{c}0.061 \pm \\
0.010\end{array}$ & $\begin{array}{c}0.208 \pm \\
0.031\end{array}$ & $\begin{array}{c}0.007 \pm \\
0.004\end{array}$ & $\begin{array}{c}0.114 \pm \\
0.013\end{array}$ & $\begin{array}{c}0.028 \pm \\
0.005\end{array}$ & $\begin{array}{c}1.386 \pm \\
0.163\end{array}$ & $\begin{array}{c}0.175 \pm \\
0.021\end{array}$ & $\begin{array}{c}0.470 \pm \\
0.019\end{array}$ & $\begin{array}{c}0.152 \pm \\
0.050\end{array}$ & $\begin{array}{c}0.011 \pm \\
0.003\end{array}$ \\
\hline & \multicolumn{12}{|c|}{ Organs: Female } \\
\hline & Lung & Heart & Thymus & $\begin{array}{c}\text { Kidney } \\
\text { L }\end{array}$ & $\begin{array}{l}\text { Adrenal } \\
\text { gland L }\end{array}$ & Snleen & $\begin{array}{c}\text { Ovary } \\
L\end{array}$ & Liver & $\begin{array}{c}\text { Pancreas } \\
\text { S }\end{array}$ & Brain & Jter & \\
\hline $\begin{array}{l}\text { Vehicle } \\
\text { control }\end{array}$ & $\begin{array}{c}0.705 \pm \\
0.050\end{array}$ & $\begin{array}{c}0.543 \pm \\
0.059\end{array}$ & $\begin{array}{c}0.216 \pm \\
0.020\end{array}$ & $\begin{array}{c}0.768 \pm \\
0.051\end{array}$ & $\begin{array}{c}0.025 \pm \\
0.008\end{array}$ & $\begin{array}{c}0.350 \pm \\
0.054\end{array}$ & $\begin{array}{c}0.085 \pm \\
0.016\end{array}$ & $\begin{array}{c}4.759 \pm \\
0.316\end{array}$ & $\begin{array}{l}0.599 \pm \\
0.023\end{array}$ & $\begin{array}{c}1.765 \pm \\
0.112\end{array}$ & $\begin{array}{c}0.744 \pm \\
0.245\end{array}$ & $\begin{array}{c}0.047 \pm \\
0.009\end{array}$ \\
\hline $\begin{array}{l}2000 \\
\mathrm{mg} / \mathrm{kg}\end{array}$ & $\begin{array}{c}0.727 \pm \\
0.042\end{array}$ & $\begin{array}{c}0.543 \pm \\
0.031\end{array}$ & $\begin{array}{c}0.205 \pm \\
0.037\end{array}$ & $\begin{array}{c}0.773 \pm \\
0.039\end{array}$ & $\begin{array}{c}0.022 \pm \\
0.009\end{array}$ & $\begin{array}{c}0.365 \pm \\
0.117\end{array}$ & $\begin{array}{c}0.079 \pm \\
0.016\end{array}$ & $\begin{array}{c}4.744 \pm \\
0.359\end{array}$ & $\begin{array}{c}0.569 \pm \\
0.088\end{array}$ & $\begin{array}{c}1.791 \pm \\
0.138\end{array}$ & $\begin{array}{c}0.607 \pm \\
0.198\end{array}$ & $\begin{array}{c}0.042 \pm \\
0.022\end{array}$ \\
\hline $\begin{array}{l}1000 \\
\mathrm{mg} / \mathrm{kg}\end{array}$ & $\begin{array}{c}0.685 \pm \\
0.068\end{array}$ & $\begin{array}{c}0.435 \pm \\
0.038\end{array}$ & $\begin{array}{c}0.234 \pm \\
0.042\end{array}$ & $\begin{array}{c}0.762 \pm \\
0.031\end{array}$ & $\begin{array}{c}0.027 \pm \\
0.015\end{array}$ & $\begin{array}{c}0.420 \pm \\
0.112\end{array}$ & $\begin{array}{c}0.083 \pm \\
0.027\end{array}$ & $\begin{array}{c}5.263 \pm \\
0.332\end{array}$ & $\begin{array}{c}0.642 \pm \\
0.095\end{array}$ & $\begin{array}{c}1.630 \pm \\
0.052\end{array}$ & $\begin{array}{c}0.708 \pm \\
0.167\end{array}$ & $\begin{array}{c}0.040 \pm \\
0.009\end{array}$ \\
\hline $\begin{array}{l}500 \\
\mathrm{mg} / \mathrm{kg}\end{array}$ & $\begin{array}{c}0.653 \pm \\
0.031\end{array}$ & $\begin{array}{c}0.494 \pm \\
0.037\end{array}$ & $\begin{array}{c}0.209 \pm \\
0.041\end{array}$ & $\begin{array}{c}0.707 \pm \\
0.038\end{array}$ & $\begin{array}{c}0.022 \pm \\
0.014\end{array}$ & $\begin{array}{c}0.390 \pm \\
0.049\end{array}$ & $\begin{array}{c}0.095 \pm \\
0.019\end{array}$ & $\begin{array}{c}4.721 \pm \\
0.439\end{array}$ & $\begin{array}{c}0.595 \pm \\
0.050\end{array}$ & $\begin{array}{c}1.603 \pm \\
0.044^{*}\end{array}$ & $\begin{array}{c}0.525 \pm \\
0.195\end{array}$ & $\begin{array}{c}0.038 \pm \\
0.008\end{array}$ \\
\hline
\end{tabular}

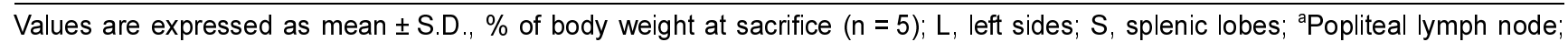
${ }^{*} p<0.05$ as compared with vehicle control.

Table 4. Necropsy findings after oral treatment of PR extracts

\begin{tabular}{|c|c|c|c|c|c|c|c|c|}
\hline \multirow[b]{2}{*}{ Group } & \multicolumn{4}{|c|}{ Male } & \multicolumn{4}{|c|}{ Female } \\
\hline & $\begin{array}{l}\text { Vehicle } \\
\text { control }\end{array}$ & $2000 \mathrm{mg} / \mathrm{kg}$ & $1000 \mathrm{mg} / \mathrm{kg}$ & $500 \mathrm{mg} / \mathrm{kg}$ & $\begin{array}{l}\text { Vehicle } \\
\text { control }\end{array}$ & $2000 \mathrm{mg} / \mathrm{kg}$ & $1000 \mathrm{mg} / \mathrm{kg}$ & $500 \mathrm{mg} / \mathrm{kg}$ \\
\hline \multicolumn{9}{|l|}{ Lung } \\
\hline Normal & $5 / 5$ & $5 / 5$ & $5 / 5$ & $5 / 5$ & $4 / 5$ & $4 / 5$ & $4 / 5$ & $5 / 5$ \\
\hline Congestion & $0 / 5$ & $0 / 5$ & $0 / 5$ & $0 / 5$ & $1 / 5$ & $1 / 5$ & $1 / 5$ & $0 / 5$ \\
\hline \multicolumn{9}{|l|}{ Thymus } \\
\hline Normal & $4 / 5$ & $5 / 5$ & $4 / 5$ & $4 / 5$ & $5 / 5$ & $5 / 5$ & $5 / 5$ & $5 / 5$ \\
\hline Atrophy & $1 / 5$ & $0 / 5$ & $1 / 5$ & $1 / 5$ & $0 / 5$ & $0 / 5$ & $0 / 5$ & $0 / 5$ \\
\hline \multicolumn{9}{|l|}{ Spleen } \\
\hline Normal & $4 / 5$ & $5 / 5$ & $3 / 5$ & $4 / 5$ & $4 / 5$ & $2 / 5$ & $4 / 5$ & $4 / 5$ \\
\hline Atrophy & $0 / 5$ & $0 / 5$ & $2 / 5$ & $1 / 5$ & $0 / 5$ & $2 / 5$ & $1 / 5$ & $0 / 5$ \\
\hline Hypertrophy & $1 / 5$ & $0 / 5$ & $0 / 5$ & $0 / 5$ & $1 / 5$ & $1 / 5$ & $0 / 5$ & $1 / 5$ \\
\hline \multicolumn{9}{|l|}{ Liver } \\
\hline Normal & $5 / 5$ & $5 / 5$ & $5 / 5$ & $4 / 5$ & $5 / 5$ & $5 / 5$ & $5 / 5$ & $5 / 5$ \\
\hline Atypical Foci & $0 / 5$ & $0 / 5$ & $0 / 5$ & $1 / 5$ & $0 / 5$ & $0 / 5$ & $0 / 5$ & $0 / 5$ \\
\hline \multicolumn{9}{|l|}{ Epididymis/Uterus } \\
\hline Normal & $5 / 5$ & $5 / 5$ & $5 / 5$ & $5 / 5$ & $4 / 5$ & $4 / 5$ & $2 / 5$ & $4 / 5$ \\
\hline Edematous changes & $0 / 5$ & $0 / 5$ & $0 / 5$ & $0 / 5$ & $1 / 5$ & $1 / 5$ & $3 / 5$ & $1 / 5$ \\
\hline \multicolumn{9}{|l|}{ Lymph node ${ }^{a}$} \\
\hline Normal & $4 / 5$ & $5 / 5$ & $5 / 5$ & $4 / 5$ & $5 / 5$ & $5 / 5$ & $5 / 5$ & $4 / 5$ \\
\hline Hypertrophy & $1 / 5$ & $0 / 5$ & $0 / 5$ & $1 / 5$ & $0 / 5$ & $0 / 5$ & $0 / 5$ & $1 / 5$ \\
\hline
\end{tabular}

Observed animals/total observed animals $(n=5) ;{ }^{a}$ Bilateral popliteal lymph node. 
brain, and uterus.

Statistical analyses. Multiple comparison tests for different dose groups were conducted. Variance homogeneity was examined using the Levene test. If the Levene test indicated no significant deviations from variance homogeneity, the obtain data were analyzed by one way ANOVA test followed by Scheffe test to determine which pairs of group comparison were significantly different. In case of significant deviations from variance homogeneity were observed at Levene test, a non-parametric comparison test, the Mann-Whitney U-Wilcoxon Rank Sum $\mathrm{W}$ test was conducted to determine the specific pairs of group comparison, which are significantly different. $\mathrm{LD}_{50}$ and $95 \%$ confidence limits were calculated by Probit method. Statistical analyses were conducted using SPSS for Windows (Release 14.0 K, SPSS Inc., USA) and a $p$-value of less than 0.05 was considered to be a significant difference. In addition, degree of clinical signs, gross and histopathological findings were subdivided into 3 degrees: 3+ Severe, 2+ moderate, 1+ slight.

\section{RESULTS}

Mortalities. No unscheduled or PR extract-treat related mortalities were detected in all dose levels

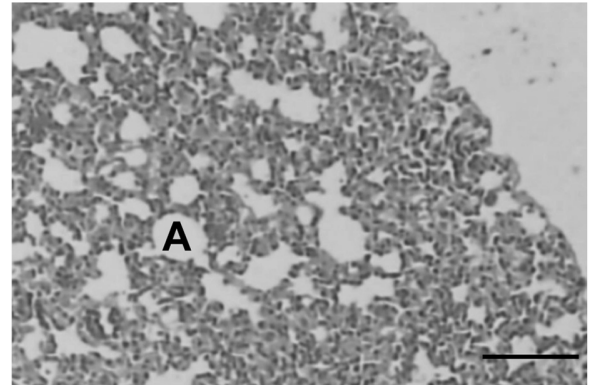

\section{Male vehicle control 01 Congestion $1+$}

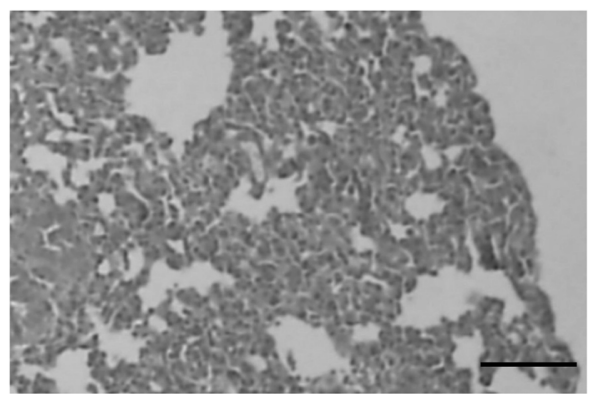

PR extracts $1000 \mathrm{mg} / \mathrm{kg}$ Male 02 Congestion $1+$

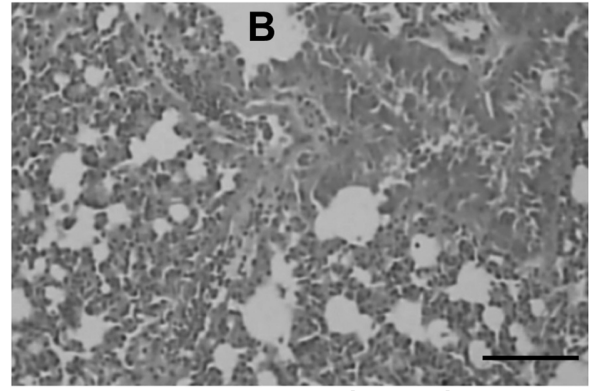

PR extracts $500 \mathrm{mg} / \mathrm{kg}$ Male 01 Congestion $1+$



Female vehicle 01

Congestion 1+



PR extracts $2000 \mathrm{mg} / \mathrm{kg}$ Female 01

Congestion $1+$



Fig. 3. Histopathological changes detected on the lung. Note that hypertrophy of alveolus with hemorrhages were randomly detected dispersed throughout the all tested groups including vehicle controls as an accidental findings. They did not show any dose dependency. A, alveolus; B, bronchiole; All Hematoxylin \& Eosin stain; Scale bars $=160 \mu \mathrm{m}$. 
tested in this study. At terminal, all of animals (5/5; $100 \%$ ) were survived in all dose levels tested including vehicle control.

Clinical signs. In this study, no PR extracts-treatment related abnormal clinical signs were observed during observation periods regardless of male and female mice.

Changes in body weights and gains. No meaningful changes on body weight and gains were detected in all dosing groups tested compared to that of vehicle control in all dose levels tested (Fig. 1 and 2, Table 1).

Changes in the organ weight. No meaningful changes on the absolute and relative organ weight of 12 principle organs were observed in all dosing groups tested compared to that of vehicle control except for significantly $(p<0.05)$ increases of absolute and relative weight of thymus in PR extract $500 \mathrm{mg} / \mathrm{kg}$-dosing male group, and significantly $(p<0.05)$ increase of relative brain weight in PR extracts $500 \mathrm{mg} / \mathrm{kg}$-dosing female group as compared with each gender of vehicle control, respectively (Table 2 and 3).

Necropsy findings. No meaningful changes on the gross findings of 12 principle organs were observed in all dosing groups tested compared to that of vehicle control except for some accidental findings such as congestion spots of lung, atrophy of thymus, spleen atrophy or hypertrophy, hypertrophy of popliteal lymph node, edematous changes of uterus, and hypertrophy of popliteal lymph nodes. They were randomly detected throughout the whole experimental groups including each gender of vehicle controls, and most of these sporadic gross findings do not showed dosedependent frequencies encounted. Atypical whiteyellow focal foci in liver were restrictly detected in one mouse of PR extracts $500 \mathrm{mg} / \mathrm{kg}$ treated male (Table 4).

Histopathological findings. No meaningful changes in the histopathological findings of 12 principle organs were observed in all dosing groups tested compared to that of vehicle control except for some sporadic acci-



Male vehicle control 05: Decreases of lymphoid cells 1+

Fig. 4. Histopathological changes detected in the thymus. Note that decreases of lymphoid cells in the cortex (as seen vacuole) was restricted to one animal $(1 / 5 ; 20 \%)$ of male vehicle control as individual sporadic findings; All Hematoxylin \& Eosin stain; Scale bars $=160 \mu \mathrm{m}$.



Male vehicle control 05: Decreases of lymphoid cells 1+

Fig. 5. Histopathological changes detected in the spleen. Note that decreases of lymphocytes in the white pulp (as seen vacuole) of spleen was restricted to one animal $(1 / 5 ; 20 \%)$ of female vehicle control as individual sporadic findings. R, red pulp; W, white pulp; All Hematoxylin \& Eosin stain; Scale bars $=160 \mu \mathrm{m}$. 


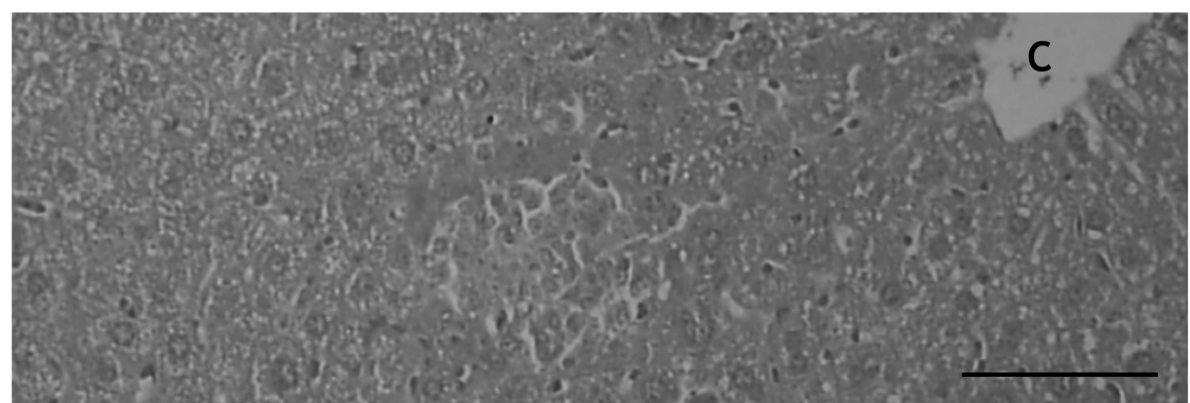

Male vehicle control 02: IF-FN 1+

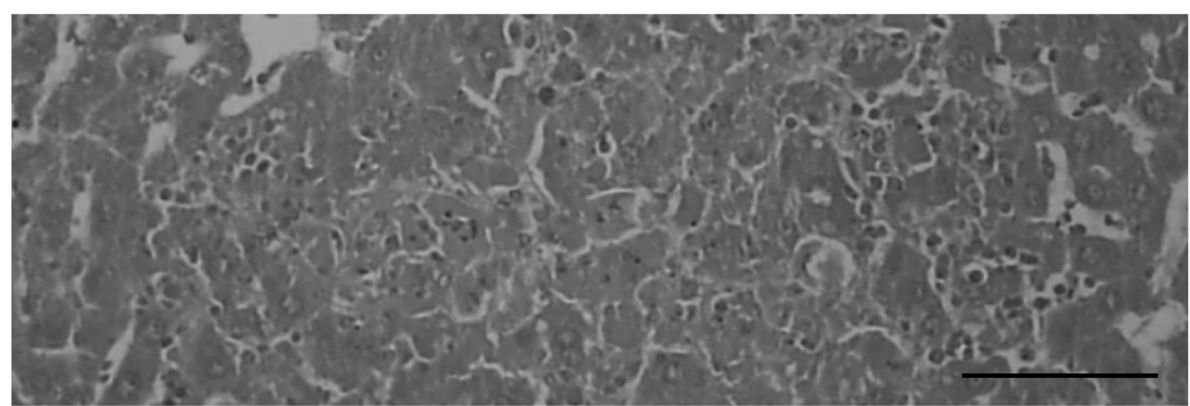

PR extracts $1000 \mathrm{mg} / \mathrm{kg}$ female 02: IF-FN 1+

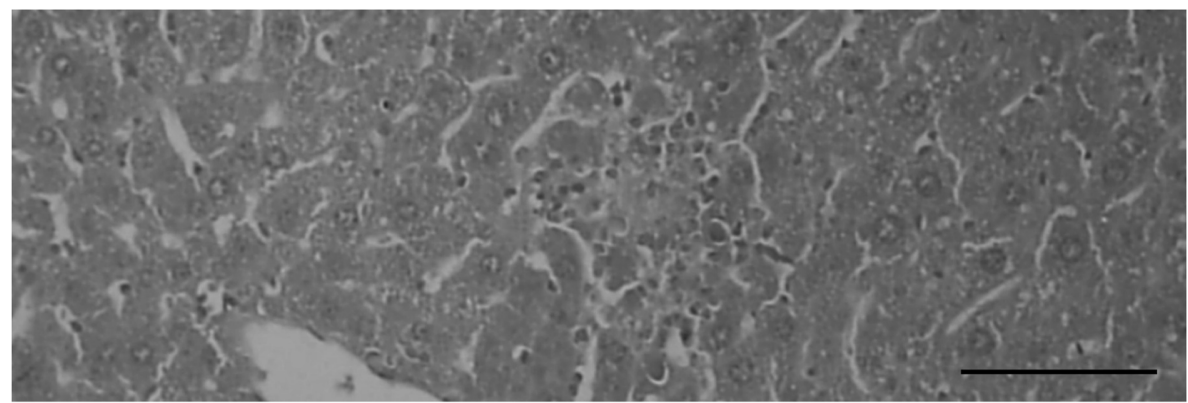

PR extracts $500 \mathrm{mg} / \mathrm{kg}$ female 01: IF-FN 1+

Fig. 6. Histopathological changes detected in the liver. Note that focal necrosis and inflammatory cell infiltration (IF-FN) in liver was restrictly observed in one mouse $(1 / 5 ; 20 \%)$ of male vehicle control, female 1000 and $500 \mathrm{mg} / \mathrm{kg}$ treated groups, respectively as an accidental findings. They were deposited between hepatic cords or as like islets. C, central vein; All Hematoxylin \& Eosin stain; Scale bars $=160 \mu \mathrm{m}$.

dental findings such as hypertrophy of lung alveolus wall as congestion (Fig. 3), depletion of lymphoid cells in the cortex of thymus (Fig. 4) and white pulp of spleen (Fig. 5), focal necrosis and inflammatory cell infiltration in liver (Fig. 6), desquamation of the uterus mucosa (Fig. 7). They were randomly detected throughout the whole experimental groups including each gender of vehicle controls, and most of these sporadic histopathological findings do not show dose-dependent frequencies encounted. In addition, somewhat increases in frequency of liver fatty changes were detected in PR extracts 2000 and $1000 \mathrm{mg} / \mathrm{kg}$ treated male and female groups (Fig. 8); only one mouse in male and female vehicle control showed slight (1+) hepatic fatty changes but four to two mice in PR extracts 2000 and $1000 \mathrm{mg} /$ $\mathrm{kg}$ treated male and female groups showed slight (1+) hepatic fatty changes with one mice in PR extracts $500 \mathrm{mg} / \mathrm{kg}$ treated groups (Table 5).

\section{DISCUSSION}

In the present study, we investigated the acute toxicity of single oral dose with PR extracts, lyophilized water extract of dried tuber of Pinellia ternata to female and male mice as a part of the safety test. PR extracts were once orally administered to female and male ICR mice at dose levels of 2000, 1000, 500 and $0 \mathrm{mg} / \mathrm{kg}$ according to the recommendation of KFDA Guidelines 


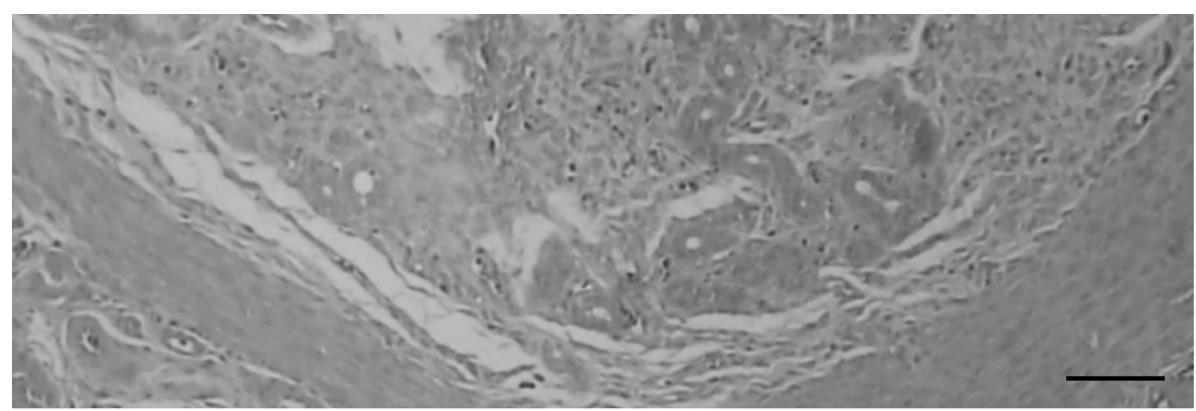

Female vehicle control 02: DM 1+

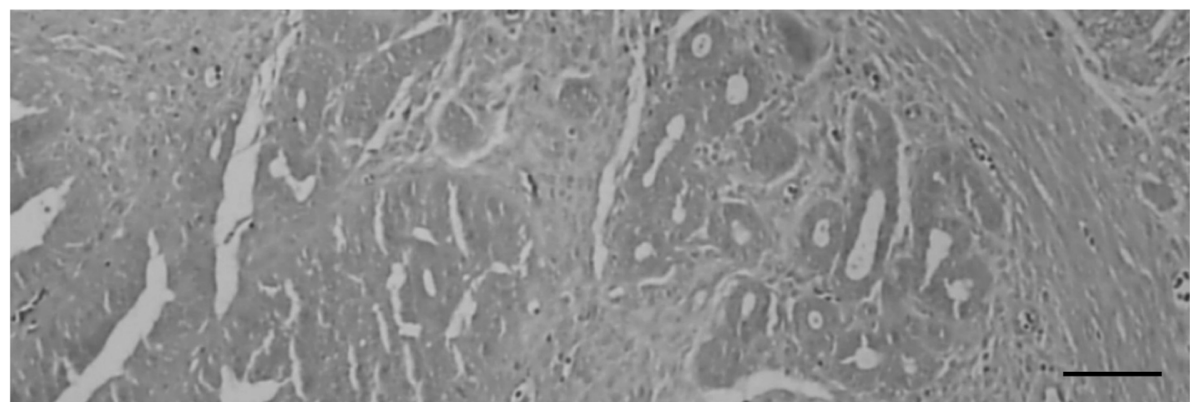

PR extracts $2000 \mathrm{mg} / \mathrm{kg}$ female 01: DM 1+



\section{PR extracts $1000 \mathrm{mg} / \mathrm{kg}$ female 02: DM 2+}

Fig. 7. Histopathological changes detected in the uterus. Note that desquamation of the uterus mucosa (DM) were detected as a process of estrus cycle. They were randomly detected throughout the whole female groups including vehicle control. All Hematoxylin \& Eosin stain; Scale bars $=160 \mu \mathrm{m}$.

(2005-60, 2005). The mortality and changes in body weight, clinical signs and gross observation were monitored during 14 days after treatment of PR extracts with organ weight and histopathology of 12 types of principle organs.

As the results, we could not find any mortality, clinical signs, changes in the body weight and gross findings and some sporadic gross findings. In addition, no meaningful changes in the organ weight and histopathology of 12 types of principle organs were detected in the present study except for dose-dependent increases of frequencies of hepatic fatty changes in PR extracts 2000 and $1000 \mathrm{mg} / \mathrm{kg}$ treated both male and female mice, and some sporadic accidental histopathological findings with dose-independent decreases of thymic absolute and relative weights in male $500 \mathrm{mg} / \mathrm{kg}$ treated mice, relative brain weights in female $500 \mathrm{mg} / \mathrm{kg}$ treated mice, respectively.

The body weight detected in this study was well corresponded to the body weight ranges of same aged normal mice as previously (Plata and Murphy, 1972; Yamaguchi et al., 1983) in all tested groups including both male and female vehicle control, respectively. It means PR extracts did not induce any harmful changes on the body weights.

In KFDA Guidelines (2005-60, 2005) and OECD Guidelines (\#423, 2001), the recommended highest dose of test materials were $2000 \mathrm{mg} / \mathrm{kg}$ or the maximum solubility, and they also recommended that in case of acute toxicity in mice, the dosage volume were 




Male vehicle control 02 Fatty changes $1+$

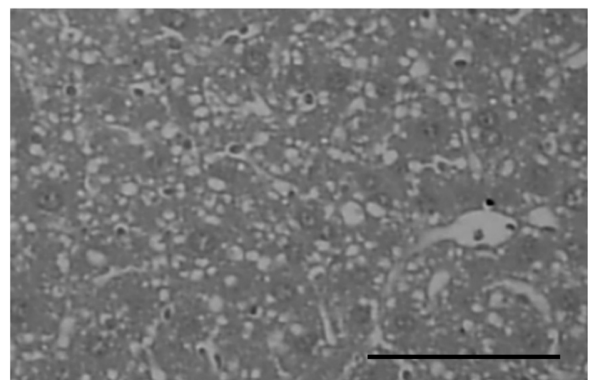

PR extracts $2000 \mathrm{mg} / \mathrm{kg}$ male 02 Fatty changes 1+

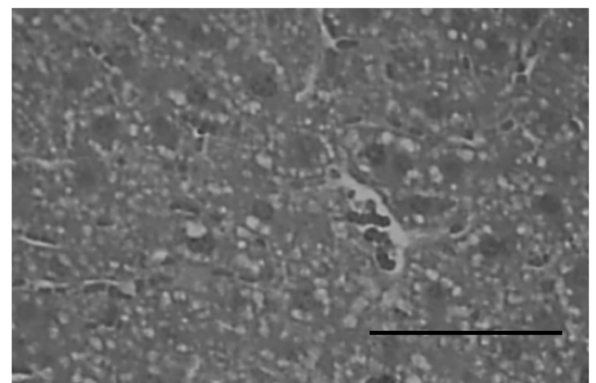

PR extracts $1000 \mathrm{mg} / \mathrm{kg}$ male 02 Fatty changes $1+$

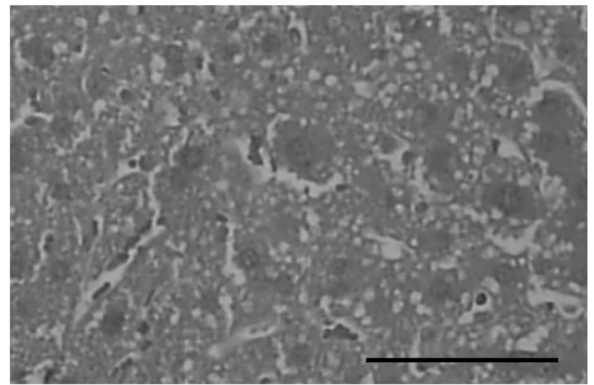

PR extracts $500 \mathrm{mg} / \mathrm{kg}$ male 02 Fatty changes $1+$

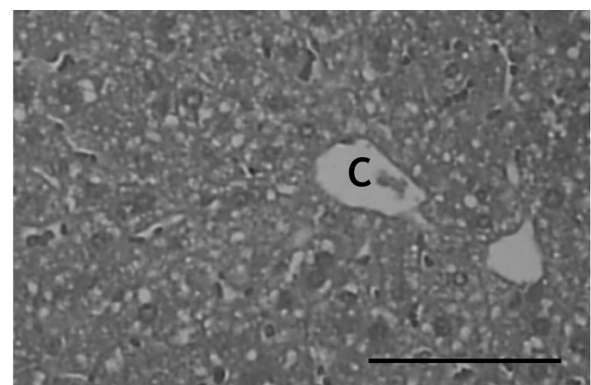

Female vehicle control 04 Fatty changes 1+

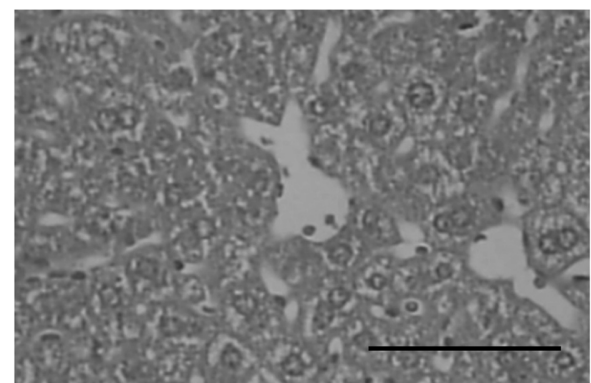

PR extracts $2000 \mathrm{mg} / \mathrm{kg}$ female 05 Fatty changes 1+

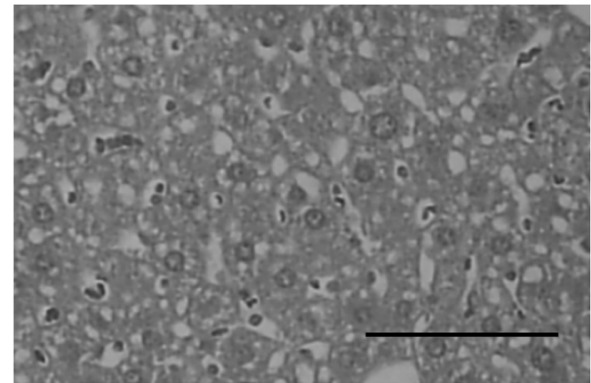

PR extracts $1000 \mathrm{mg} / \mathrm{kg}$ female 03 Fatty changes $1+$

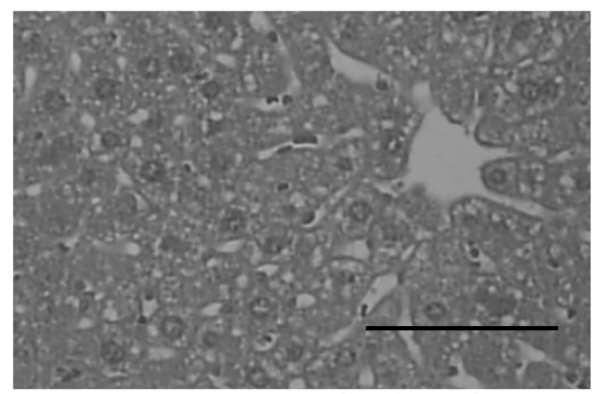

PR extracts $500 \mathrm{mg} / \mathrm{kg}$ female 03

Fatty changes $1+$

Fig. 8. Histopathological changes detected in the liver fatty changes. Note that increases of frequency of liver fatty changes were detected in PR extracts 2000 and $1000 \mathrm{mg} / \mathrm{kg}$ treated male and female groups; only one mouse in male and female vehicle control showed slight (1+) fatty changes but they were more frequently detected in PR extracts 2000 and $1000 \mathrm{mg} / \mathrm{kg}$ treated male and female groups. They were deposited as lipid droplet in hepatocytes and/or induced hypertrophy of hepatocytes around central veins; C, central vein; All Hematoxylin \& Eosin stain; Scale bars $=160 \mu \mathrm{m}$.

below $20 \mathrm{ml} / \mathrm{kg}$ in case of clear soluble materials but $10 \mathrm{ml} / \mathrm{kg}$ in suspensions. In the present study, the high- est dose of PR extracts was selected as $2000 \mathrm{mg} / \mathrm{kg}$ because PR extracts have been used as folk medicine 
Table 5. Histopathological findings after oral treatment of PR extracts

\begin{tabular}{|c|c|c|c|c|c|c|c|c|}
\hline \multirow[b]{2}{*}{ Group } & \multicolumn{4}{|c|}{ Male } & \multicolumn{4}{|c|}{ Female } \\
\hline & $\begin{array}{l}\text { Vehicle } \\
\text { control }\end{array}$ & $2000 \mathrm{mg} / \mathrm{kg}$ & $1000 \mathrm{mg} / \mathrm{kg}$ & $500 \mathrm{mg} / \mathrm{kg}$ & $\begin{array}{l}\text { Vehicle } \\
\text { control }\end{array}$ & 2000 mg/kg & $1000 \mathrm{mg} / \mathrm{kg}$ & $500 \mathrm{mg} / \mathrm{kg}$ \\
\hline \multicolumn{9}{|l|}{ Lung } \\
\hline Normal & $4 / 5$ & $5 / 5$ & $4 / 5$ & $3 / 5$ & $4 / 5$ & $4 / 5$ & $4 / 5$ & $5 / 5$ \\
\hline Congestion & $1 / 5$ & $0 / 5$ & $10 / 5$ & $2 / 5$ & $1 / 5$ & $1 / 5$ & $1 / 5$ & $0 / 5$ \\
\hline \multicolumn{9}{|l|}{ Thymus } \\
\hline Normal & $4 / 5$ & $5 / 5$ & $5 / 5$ & $5 / 5$ & $5 / 5$ & $5 / 5$ & $5 / 5$ & $5 / 5$ \\
\hline $\mathrm{DE}^{*}$ & $1 / 5$ & $0 / 5$ & $0 / 5$ & $0 / 5$ & $0 / 5$ & $0 / 5$ & $0 / 5$ & $0 / 5$ \\
\hline \multicolumn{9}{|l|}{ Spleen } \\
\hline Normal & $5 / 5$ & $5 / 5$ & $5 / 5$ & $5 / 5$ & $4 / 5$ & $5 / 5$ & $5 / 5$ & $5 / 5$ \\
\hline $\mathrm{DE}^{*}$ & $0 / 5$ & $0 / 5$ & $0 / 5$ & $0 / 5$ & $1 / 5$ & $0 / 5$ & $0 / 5$ & $0 / 5$ \\
\hline \multicolumn{9}{|l|}{ Liver } \\
\hline Normal & $4 / 5$ & $1 / 5$ & $2 / 5$ & $4 / 5$ & $4 / 5$ & $2 / 5$ & $3 / 5$ & $3 / 5$ \\
\hline IF-FN ${ }^{*}$ & $1 / 5$ & $0 / 5$ & $0 / 5$ & $0 / 5$ & $0 / 5$ & $0 / 5$ & $1 / 5$ & $1 / 5$ \\
\hline Fatty changes & $1 / 5$ & $4 / 5$ & $3 / 5$ & $1 / 5$ & $1 / 5$ & $3 / 5$ & $1 / 5$ & $1 / 5$ \\
\hline \multicolumn{9}{|l|}{ Epididymis/Uterus } \\
\hline Normal & $5 / 5$ & $5 / 5$ & $5 / 5$ & $5 / 5$ & $3 / 5$ & $4 / 5$ & $2 / 5$ & $5 / 5$ \\
\hline $\mathrm{DM}^{*}$ & $0 / 5$ & $0 / 5$ & $0 / 5$ & $0 / 5$ & $2 / 5$ & $1 / 5$ & $3 / 5$ & $0 / 5$ \\
\hline
\end{tabular}

Observed animals/total observed animals $(n=5)$; ${ }^{a}$ Bilateral popliteal lymph node; * Abbreviations: DE, decreases of lymphoid cells; IF-FN, inflammatory cell infiltration with necrosis; DM, desquamation of mucosa.

and ingredients of medicinal food for long times and no revealed toxicological data was available, base on the recommendation of KFDA $(2005-60,2005)$ and OECD Guidelines (\#423, 2001), and treated in a volume of $20 \mathrm{ml} / \mathrm{kg}$ using distilled water as vehicle because PR extracts were clearly dissolved upto $100 \mathrm{mg} / \mathrm{ml}$, at least in the present study.

Significant $(p<0.05)$ decreases of absolute and relative thymic weights in PR extracts $500 \mathrm{mg} / \mathrm{kg}$ male mice, and relative brain weights in PR extracts $500 \mathrm{mg} /$ $\mathrm{kg}$ male mice were considered as not PR extracts-treatment related changes because they were not showed dose-dependency with no changes on the histopathological profiles, and the other organ weights measured in this study well corresponded to the normal mice organ weight ranges as previously (Plata and Murphy, 1972; Yamaguchi et al., 1983).

The depletion of lymphoid cells in thymus detected in one animal of male vehicle control was considered as a result of physiological involution (Banks, 1986), and splenic white pulp lymphoid cells depletion detected in one female vehicle control mouse is also considered as individual changes. Edematous changes in the uterus at gross findings and related desquamation of uterus mucosa at histopathological observation in the present study were also considered as a result of differences of physiological estrus cycles (Banks, 1986; Pineda, 1989) not PR extracts-dosing relative changes. They were also detected in vehicle control. The atypical yellow foci detected in one mouse of PR extract $500 \mathrm{mg} / \mathrm{kg}$ treated group revealed as slight fatty changes at histopathologi- cal observations.

The dose-dependent increases of frequencies of hepatic fatty changes detected in PR extracts 2000 and $1000 \mathrm{mg} / \mathrm{kg}$ treated both male and female mice, considered as direct evidences that PR extracts can be induced hepatotoxicity. The fatty changes in hepatocytes have been regarded as mild damages of liver (Maclachlan and Cullen, 1995). However, in the previous PR extracts 4 weeks repeated oral dose toxicity test in rat (Lee et al., 2003), the possibilities of hepatotoxicity did not suggested. Therefore, it is considered that the toxicity of PR extracts have species-specific patterns.

Congestion spots of lung, atrophy of thymus, spleen atrophy or hypertrophy, hypertrophy of popliteal lymph node and hypertrophy of popliteal lymph nodes detected as gross findings, and hypertrophy of lung alveolus wall as congestion and focal necrosis and inflammatory cell infiltration in liver detected as histopathological findings were considered as accidental findings and they were not considered as PR extracts-treatment related abnormal gross or histopathological findings because they were restricted in some individual animals and most of them, also observed in male and female vehicle controls. . In addition, most of them were rarely observed in normal mice (Lee et al., 2005, 2006).

Although the Hodge and Sterner (1949) classify as non toxic materials those $\mathrm{LD}_{50}$ were $5000 \sim 15000 \mathrm{mg} / \mathrm{kg}$ and the materials those $\mathrm{LD}_{50}$ were $500 \sim 5000 \mathrm{mg} / \mathrm{kg}$ also classified as relatively low toxic (Class III) by US Environmental Protection Agency (OPPTS 870.100, 
1998), recently Notified Guidelines by KFDA (2005-60, 2005) and OECD Guidelines (\#423, 2001) recommended that the highest oral dose of test materials was $2000 \mathrm{mg} / \mathrm{kg}$. The $L D_{50}$ and ALD in mice after single oral dose of PR extracts were detected over $2000 \mathrm{mg} / \mathrm{kg}$ in both male and female in the present study. However, more than $1000 \mathrm{mg} / \mathrm{kg}$ of PR extracts treatment could be induced slight hepatotoxicity in mice, the fatty changes.

\section{REFERENCES}

Banks, W.J. (1986). Female reproductive system in Applied veterinary histology (Banks, W.J. Ed.). Williams \& Wilkins, Baltimore, pp. 330-347, 506-526.

Chen, D., Wu, C.F., Huang, L. and Ning, Z. (2002). Effect of the aqueous extract of xiao-ban-xia-tang on gastric emptying in mice. Am. J. Chin. Med., 30, 207-214.

Chen, Z.X., Zhang, S.J., Lao, S.X., Hu, H.T., Zhang, C.Y., Guan, S.H. and Gu, Y.L. (2005). He Jie Tang in the treatment of chronic hepatitis B patients. World J. Gastroenterol., 11, 6638-6643.

Chung, T.W., Koo, B.S., Choi, E.G., Kim, M.G., Lee, I.S. and $\mathrm{Kim}$, C.H. (2006). Neuroprotective effect of a chuk-mesun-dan on neurons from ischemic damage and neuronal cell toxicity. Neurochem. Res., 31, 1-9.

Dourish, C.T. (1987). Effects of drugs on spontaneous motor activity in experimental psychopharmacology (Greenshaw, A.J. and Dourish, C.T. Eds.). Humana Press, Clifton, pp. 325-334

Hodge, H.C. and Sterner, J.H. (1949). Tabulation of toxicity classes. Am. Ind. Hyg. Q., 10, 93.

Ho, S.T. and Liu, T.S. (1975). Studies on Pinellia ternate Breitenbach. Report I. Antiemetic action of Pinellia ternata Breitenbach and its components. Taiwan Yao Hsueh Tsa Chih, 27, 41-46

Irwin, S. (1968). Comprehensive observational assessment: la. A systemic, quantitative procedure for assessing the behavioral and physiological state of the mouse, Psychopharmacology, 13, 222-257.

Katagiri, F., Inoue, S., Sato, Y., Itoh, H. and Takeyama, M. (2004). Comparison of the effects of Sho-hange-ka-bukuryo-to and Nichin-to on human plasma adrenocorticotropic hormone and cortisol levels with continual stress exposure. Biol. Pharm. Bull., 27, 1679-1682.

Kim, Y.J., Shin, Y.O., Ha, Y.W., Lee, S., Oh, J.K. and Kim, Y.S. (2006). Anti-obesity Effect of Pinellia ternata Extract in Zucker Rats. Biol. Pharm. Bull., 29, 1278-1281.

Koo, B.S., Choi, E.G., Park, J.B., Cho, C.H., Chung, K.H. and Kim, C.H. (2005). Neuroprotective effect of Chuk-Me-SunDan on NMDA- and AMPA-evoked nitric oxide synthase activity in mouse brain. Immunopharmacol. Immunotoxicol., 27, 499-514.

Korea Food and Drug Administration. Testing Guidelines for Safety Evaluation of Drugs (Notification No. 2005-60, issued by the Korea Food and Drug Administration on October 21, 2005), 2005

Kurata, K., Takaaki, T., Ye, Y., Kaoru, K., Kiyotaka, K., Kunio,
T., Kazuo, W. and Yoshiki, N. (1998). Quantitative analysis of anti-emetic principle in the tubers of Pinellia ternata by enzyme immunoassay. Plant Med., 64, 645-648

Lee, H.S., Lee, I.G. and Ku, S.K. (2006). Single oral dose toxicity study of water extracts of Picrorrhiza Rhizoma in mice. J. Toxicol. Pub. Health, 22, 117-126.

Lee, J.E., Kim, H.J., Choi, E.K., Chai, H.Y., Yun, Y.W., Kim, D.J., Nam, S.Y., Lee, B.J., Ahn, B.W., Kang, H.G. and Kim, Y.B. (2003). Four-week repeated-dose toxicity study on pinellia extract. Korean J. Lab. Anim. Sci., 19, 127-141.

Lee, J.H., Yang, K.J., Shin, H.D., Park, B.R., Son, C.W., Jang, H.J., Park, D.C., Lee, H.S. amd Ku, S.K. (2005). Single subcutaneous dose toxicity of $\operatorname{Polycan}^{\circledR}$, a $\beta$-glucan originated from Aureobasidium in mice. Lab. Anim. Res., 21, 299-305.

Lee, S.B., Kim, D.J., Kim, K.J., Jeon, B.H. and Woo, W.H. (1993). Study on the cytotoxic effect of Pinelliae Tuber extracts according to Prebrewed methods. J. Won Kwang Oriental Med., 3, 65-80.

Li, Y.L., Gan, G.P., Zhang, H.Z., Wu, H.Z., Li, C.L., Huang, Y.P., Liu, Y.W. and Liu, J.W. (2007). A flavonoid glycoside isolated from Smilax china $\mathrm{L}$. rhizome in vitro anticancer effects on human cancer cell lines. J. Ethnopharmacol., $113,115-124$

Maclachlan, N.J. and Cullen, J.M. (1995). Liver, biliary system, and exocrine pancreas in Thomson's Special Veterinary Pathology (Carlton, M.W. and McGavin, M.D. Eds.). Mosby, St. Louis, pp. 81-115.

Maki, T., Takahashi, K. and Shibata, S. (1987). An antiemetic principle of Pinellia ternata Tuber. Plant Med., 53, 410-414

Organization for Economic Co-Operation and Development (Ed.). OECD guideline (423) for testing of chemicals acute oral toxicity-acute toxic class method. 2001.

Park, J.H. and Seo, B.I. (2000). A philological study on poisoning of Pinelliae Rhizoma. J. East-West Med., 25, 47-55.

Pineda, M.H. (1989). Female reproductive system in Veterinary endocrinology and reproduction (McDonald, L.E. and Pineda, M.H. Eds.). Lea \& Febiger, Philadelphia, pp. 303354.

Plata, E.J. and Murphy, W.H. (1972). Growth and haematologic properties of the BALB/wm strain of inbred mice. Lab. Anim. Sci., 22, 712-720.

Shi, Y.L., Xu, Y.F. and Zhang, H. (1994). Effect of Pinellia ternata lectin on membrane currents of mouse motor nerve terminals. Sci. China B., 37, 448-453.

U.S. Environmental Protection Agency. Health Effects Test Guidelines OPPTS 870.100, Acute Toxicity Testing Background. US EPA August, Washington, USA (1998).

Yamaguchi, C., Fujita, S., Obara, T. and Ueda, T. (1983). Effects of room temperature on reproduction, body weight and organ weights, food and water intakes, and hematology in mice. Exp. Anim., 32, 1-11.

Yang, H.C., Chung, M.H. and Chang, I.M. (2000). Study on the micronucleus test of Pinelliae Tuber Extracts according to Prebrewed methods. Poster presentation PB13. Kor. J. Pharmacogn., 21, 258-259.

Zhao, Y.J., Ji, Z.Q., Zhang, X.N., Zhang, Y.Y., Wu, J.F., Fang, $X$ and Yue, W. (2005). Effect of Rhizoma pinelliae on vomiting in minks. Zhongguo Zhong Yao Za Zhi, 30, 277-279. 